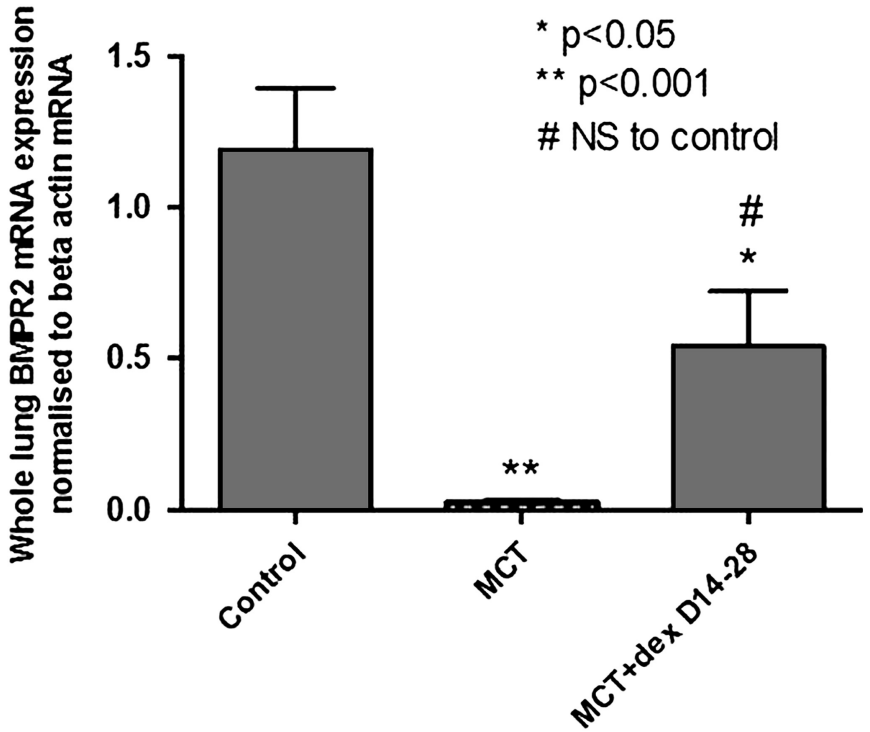

Abstract S152 Figure 1 Effects of monocrotaline and day 14-28 dexamethasone on whole-lung BMPR2 expression by qRT-PCR. migration) were added to the upper chamber and either leakage of FITC-albumin or transmigration of leukocytes into the lower chamber assessed over time. Using a flow-based model, TNF $\alpha$ (4 h) or TGF $\beta 1$ (24 h) stimulated HPAEC were seeded into Ibidi slides and leukocyte-endothelial interactions visualised, recorded and quantified. In vivo, permeability was assessed by measuring Evans blue leakage into the pulmonary vasculature in endothelial restricted BMPR-II deficient mice. Myeloperoxidase (MPO) in the lungs was assessed as a measure of leukocyte infiltration.

Results Leakage of FITC-albumin through HPAEC with reduced BMPR-II expression was significantly increased compared to mocktransfected HPAEC. Under static and flow conditions, leukocyte transmigration was greatly increased through HPAEC with reduced BMPR-II expression following TNF $\alpha$ or TGF $\beta 1$ stimulation. This facilitated transmigration following loss of BMPR-II could be blocked by pharmacological intervention of CXCR2.

Conclusions Our data suggest a novel role for BMPR-II in dampening inflammatory signals in the pulmonary vasculature and that loss of BMPR-II in the endothelial layer of the pulmonary vasculature may lead to heightened susceptibility to inflammation-induced tissue damage. We speculate this may be a key mechanism involved in the initiation of the disease in heritable PAH that result from defects in BMPR-II expression. down-regulation $(p<0.01)$ following monocrotaline, which was significantly increased following day 14-28 dexamethasone treatment in whole lung $(\mathrm{p}<0.05)$ (Abstract S152 Figure 1). Cellular $B M P R 2$ was also increased following in vitro treatment of control pulmonary artery smooth muscle cells (PASMC) with $\times 10^{-8}$ molar dexamethasone $(p<0.05)$, but not in PASMC isolated from pulmonary hypertensive rats. Dexamethasone $\left(\times 10^{-8}\right.$ and $10^{-7}$ molar $)$ also reduced proliferation of PASMC isolated from both control and pulmonary hypertensive rats ( $\mathrm{p}<0.05$ for both doses).

Conclusion $\mathrm{PAH}$ in this well-characterised experimental model can be reversed by dexamethasone, and survival is improved. In this model, mechanisms may involve reduction of IL-6-expressing inflammatory cells, reduced proliferation of vascular smooth muscle cells, and restoration of pulmonary BMPR2 expression may be important.

\section{S153 BONE MORPHOGENETIC PROTEIN RECEPTOR-II REGULATES PULMONARY ARTERY ENDOTHELIAL CELL BARRIER FUNCTION: RELEVANCE TO HERITABLE PULMONARY ARTERIAL HYPERTENSION}

doi:10.1136/thx.2010.150953.4

${ }^{1} \mathrm{~V}$ J Burton, ${ }^{1} \mathrm{~L}$ I Ciuclan, ${ }^{1} \mathrm{~A} M$ Holmes, ${ }^{2} \mathrm{D}$ Rodman, ${ }^{1} \mathrm{C}$ Walker, ${ }^{1} \mathrm{D}$ C Budd. ${ }^{1}$ Respiratory Disease Area, Novartis Institutes for BioMedical Research, Wimblehurst Road, Horsham, West Sussex RH12 5AB, UK; ${ }^{2}$ Respiratory Translational Medicine, Novartis Institutes for BioMedical Research 220 Massachusetts Avenue, 3rd floor, Cambridge, MA 02139, USA

Background Mutations in bone morphogenetic protein receptor II (BMPR-II) have been shown to underlie most heritable cases of Pulmonary arterial hypertension (PAH). However, less than half the individuals who harbour mutations develop the disease. This fact has lead to speculation that the genetic defect combined with an additional trigger, such as inflammation, may be required for the disease to be manifested.

Aim To define the role of BMPR-II in regulating the barrier function of pulmonary artery endothelial cells (PAEC).

Methods In vitro, BMPR-II expression was reduced in HPAEC using siRNA and cells were seeded onto transwell filters. FITC-labelled albumin (to assess permeability) or leukocytes (to assess leukocyte

\section{A. Pulmonary vascular leakage}

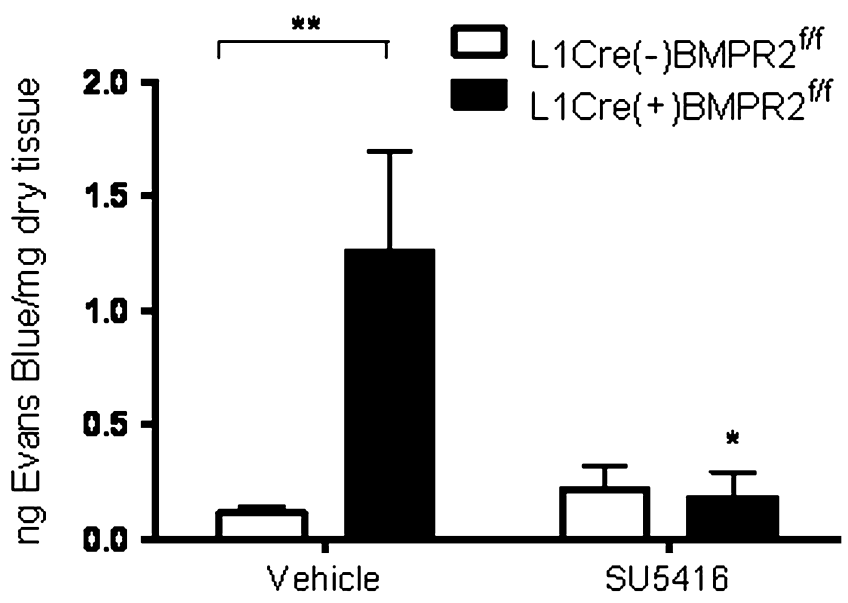

\section{B. Leukocyte infiltration}

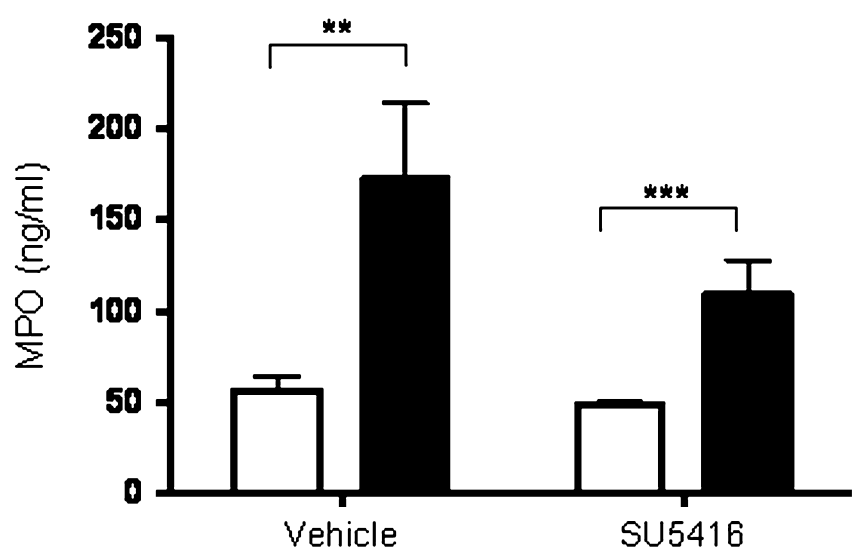

Abstract S153 Figure 1 